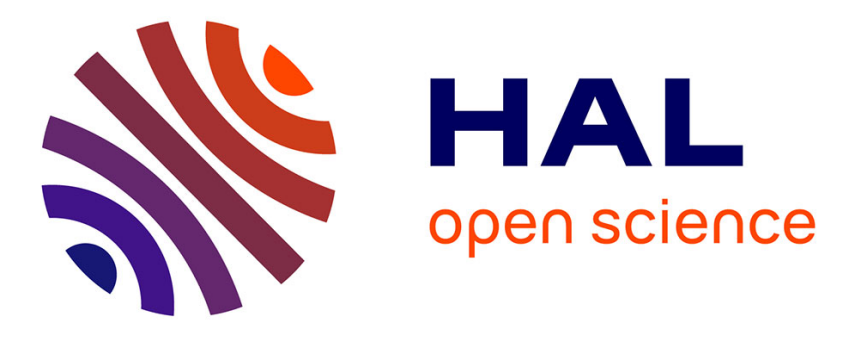

\title{
Registration of free-breathing abdominal 3D contrast-enhanced CT
}

Blandine Romain, Veronique Letort, Olivier Lucidarme, Florence d'Alché-Buc, Laurence Rouet

\section{> To cite this version:}

Blandine Romain, Veronique Letort, Olivier Lucidarme, Florence d'Alché-Buc, Laurence Rouet. Registration of free-breathing abdominal 3D contrast-enhanced CT. 4th International Workshop on Abdominal Imaging. Computational and Clinical Applications, Held in Conjunction with MICCAI 2012, Oct 2012, Nice, France. pp.274-282, 10.1007/978-3-642-33612-6_29 . hal-00780707

\section{HAL Id: hal-00780707 https://hal.science/hal-00780707}

Submitted on 24 Jan 2014

HAL is a multi-disciplinary open access archive for the deposit and dissemination of scientific research documents, whether they are published or not. The documents may come from teaching and research institutions in France or abroad, or from public or private research centers.
L'archive ouverte pluridisciplinaire HAL, est destinée au dépôt et à la diffusion de documents scientifiques de niveau recherche, publiés ou non, émanant des établissements d'enseignement et de recherche français ou étrangers, des laboratoires publics ou privés. 


\title{
Registration of free-breathing abdominal 3D contrast-enhanced CT
}

\author{
Blandine Romain ${ }^{1,2,5}$, Véronique Letort ${ }^{2}$ Olivier Lucidarme $^{4}$, Florence \\ d'Alché-Buc ${ }^{3,5}$, and Laurence Rouet ${ }^{1}$ \\ ${ }^{1}$ Medisys, Philips Research, Suresnes,France \\ 2 MAS Lab, Ecole Centrale Paris, Chatenay-Malabry, France \\ ${ }^{3}$ INRIA-Saclay, LRI CNRS 8623, Orsay, France \\ ${ }^{4}$ AP-HP, Hospital La Pitié-Salpétrière, Paris, France \\ ${ }^{5}$ IBISC, University of Evry, Evry, France
}

\begin{abstract}
CT perfusion imaging is used for the follow-up of abdominal tumors. A specificity of our work is that patients are breathing freely during image acquisition ( 5 minutes). We propose an automatic 3D image registration to compensate respiratory motion. The registration is computed in two main steps: global translation in the z-direction and 3D multiresolution blockmatching. Within this algorithm, the choice of similarity measure largely determines the algorithm robustness in presence of intensity shifts due to contrast diffusion. We exploit a modified entropy-based similarity measure to improve the quality of registration. We also propose two relevant criteria allowing to quantify the registration quality: one based on the gradients of image volumes of differences and one based on the smoothness of enhanced-intensity curves.
\end{abstract}

Keywords: free-breathing, spatio-temporal registration, DCE-CT, evaluation criteria

\section{Introduction}

Functional imaging has gained attention for oncology therapy. It is based on the acquisition of time sequences combined with contrast injection. Long acquisitions (up to 5 minutes) allow to study the complete dynamics of diffusion, including the effects of tissue permeability. However, it is possible to get relevant information only if data are correctly registered. Two main approaches are possible to deal with respiratory motion, sequential breath hold [1] or free-breathing. In the present study, free breathing is selected, since a less stressed patient usually provides sequences with more regular motion. The current study focuses on tumors in liver or kidney using dynamic enhanced CT.

Methods for registration of images acquired with free-breathing are being developed essentially for contrast-enhanced MRI. Wollny et al. [7] exploit the quasi periodicity within myocardial perfusion and $\mathrm{Li}$ et al. [2] explicitly estimate the respiratory cycle within abdominal perfusion. In both cases, an important assumption is a high time resolution. 
In $\mathrm{CT}$, it is also possible to have high time resolution, but this is combined with a short time of acquisition (2 minutes) [4], and this does not allow to extract permeability. For CT perfusion, with the objective of extracting adequate data for functional analysis, a longer time of acquisition is required which implies, mostly for dose considerations, a low time resolution. The second constraint is a limited imaging field of view in z-direction. To the best of our knowledge, no previous studies have investigated image registration on free-breathing with abdominal dynamic contrast-enhanced CT (DCE-CT) for long acquisitions.

In that context, the aim of this paper is, in a first part, to propose the complete pipeline of the intensity-based methods that we have proposed to compensate free respiratory motion. The second main contribution of this study is the definition and evaluation of a dedicated similarity measure adapted from the difference entropy (DE), which is compared to the classically used DE [3]. Finally, we propose the definitions of a temporal and a spatial criteria, in order to objectively evaluate the quality of the registration and to compare the results obtained when testing the different similarity measures.

\section{MATERIALS AND METHODS}

\subsection{Data acquisition}

39 data acquisitions were performed with a 256 slices CT (Brilliance iCT 256, Philips Healthcare, The Netherlands) on 14 patients. All patients gave informed consent. The dynamic CT protocol consists in acquiring 48 volumes every 2.5 seconds then 18 volumes every 10 seconds $(80 \mathrm{kV}, 80 \mathrm{mAS}$, rotation time of 0.33 seconds, dose of iodine $80 \mathrm{cc}$ ). With $8 \mathrm{~cm}$ detector coverage in z-direction, the CT scanner allows for $5.5 \mathrm{~cm}$ effective z-coverage in a single rotation with a 3D axial cone beam correction. This coverage is large enough to keep most of the lesion in image volume. In the axial plane, data are reconstructed with a pixel size of $0.68 \mathrm{~mm} \times 0.68 \mathrm{~mm}$ [5]. Reconstructed volumes are expressed in Hounsfield Units (HU). Image intensities can thus range from -1024 (air) to $2000 \mathrm{HU}$ (bone).

\subsection{Spatio-Temporal registration}

The main challenges for registration in these conditions are:

- Limited volume height: data acquisition covers about $6 \mathrm{~cm}$ in z-direction, while the amplitude of respiratory motion may reach 2 to $3 \mathrm{~cm}$, in the same z-direction. This means that the whole liver or kidneys are not imaged. Consequently, full organ tracking is not possible and local approaches have to be used.

- Contrast agent injection: intensities of a given tissue vary with time (figure 1). The choice of a similarity measure which is robust to contrast-induced intensity variations is essential in our method. 
- Free breathing and low time resolution: since patients breath freely, a first consequence is that acquisitions correspond to different instants in respiratory cycle and a prediction using a respiratory model is not possible. A second consequence is the existence of strong intensity variations between two consecutive volumes due to contrast arrival and low time resolution ( 2.5 or 10 seconds). In such a context, the problem of intensity differences is present even between successive pairs of volumes $\left(I_{t}\right.$ onto $\left.I_{t-1}\right)$ and registering these pairs of volumes would lead to unnecessary errors accumulation. So, it is preferable to align all volumes onto a common reference volume $I_{r}$. Since the majority of image volumes are enhanced, an intuitive solution would be to choose a reference volume $I_{r}$ with contrast $(t \approx 30)$. However, the assumption that the tumor is well centered can only be done in the first volume, which is why we selected it as the reference volume $I_{r}$.
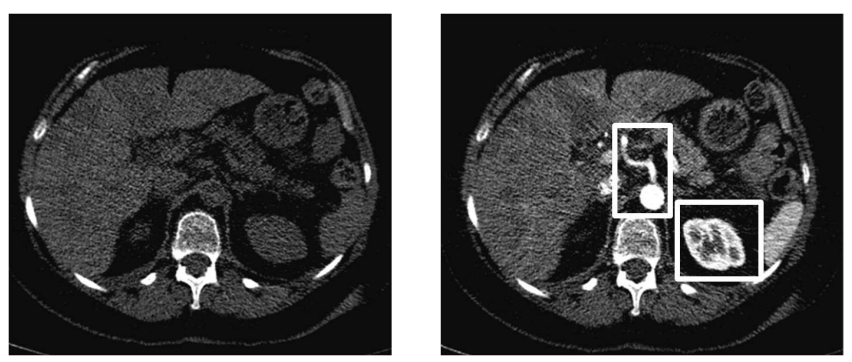

Fig. 1. Image data at time 0 (left) and 15 seconds after injection (right). On the right figure: kidney is enhanced (white square) and aorta with hepatic artery are enhanced (white rectangle, middle).

In order to deal with these challenges, we present an adapted spatio-temporal registration pipeline. Each reconstruction provides a dynamic sequence $D S_{I}=$ $\left(I_{t}, t \in(1, \ldots T)\right)$, where $I_{t}$ is a $3 \mathrm{D}$ image acquired at time $t$ and $T$ is the number of acquisition times.

Our registration approach involves two main steps, followed by a step of regularization and warping:

1. Global z-translation : since the main motion is in the z-direction, the first step consists in a global evaluation of the z-translation. To improve this initial step, thresholds are applied to exclude air $(\sim-1024 \mathrm{HU})$ and intense contrasts $(\geq 600 \mathrm{HU})$, such as the contrast of the static aorta in z-direction. We considerer all pixels which intensity is between $-800 \mathrm{HU}$ and $600 \mathrm{HU}$. Since we are considering one global translation for the whole masked volume at this point, we verified that the local contrast diffusion was not an important disturbance, and that the sum of square of differences (SSD) was the most appropriate metric (compared to entropy and mutual information, results not shown). 
2. Multi-resolution blockmatching [6]: initialized by the z-translation found in the first step, this 3D registration method is computed with block sizes of $\left(11 \times 11 \times 7 \mathrm{~mm}^{3}\right)$. It provides, for each block, motion vectors corresponding to translations in $\mathrm{x}, \mathrm{y}$ and $\mathrm{z}$ directions.

3. Regularization: a regularization step is necessary to smooth the motion vector fields. A gaussian filter is computed in $3 \mathrm{D}(\sigma=1.2)$.

4. Warping: warping consists in reconstructing new image sequences $\left(J_{1}, \ldots J_{T}\right)$ with respiratory motion compensation. A trilinear interpolation is used.

\subsection{Masking}

In order to improve the algorithm robustness and its computing efficiency, masked volumes are computed. For each volume, a mask is defined by excluding spine, which have a different motion from the rest of the abdomen, and background, which does not give useful information.

Our mask consists of two 3D zones with z-translation invariance. These two zones can therefore be represented in an axial plane of arbitrary z-value.

On each axial plane, a threshold of $500 \mathrm{HU}$ is first applied to extract the bones: the largest connected component is the spine. Similarly, the largest connected component of pixels of intensity values lower than $-500 \mathrm{HU}$ are considered to belong to the background (figure 2).

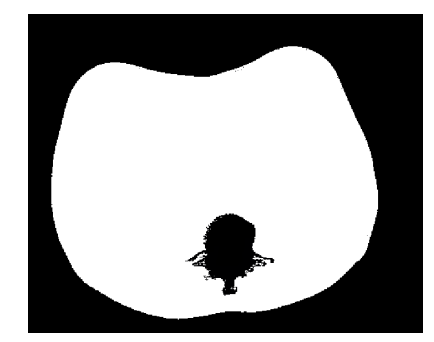

Fig. 2. Mask example: white zone is the valid region, excluding spine and background.

\subsection{Similarity measures for multi-resolution blockmatching}

To locally register the volumes $I_{r}$ and $I_{t}$, our approach is based on a similarity measure which is potentially robust to intensity variations, namely DE. The entropy $H$ of a discrete random variable $X$ with probability mass function $p(X)$ is defined as:

$$
H(X)=\mathbb{E}(-\log p(X))
$$

The difference between $I_{r}$ and $I_{t}$, noted $I_{r-t}$, is used to evaluate DE. The classical definition is based on the normalized histogram hist $\left(I_{r-t}\right)$ of $I_{r-t}$ with $B$ bins:

$$
D E=-\sum_{j=1}^{B} h i s t\left(I_{r-t}\right)_{j} * \log \left(h i s t\left(I_{r-t}\right)_{j}\right)
$$


DE is classically used for registration, in particular in contrast enhanced echocardiography [3].

However, it is known that the quality of registration is very dependent on the number of bins $B$. So, our approach is to approximate DE to have a more dedicated and robust similarity measure. We can define $H$ directly in the image domain $\Omega$ as:

$$
H=\frac{1}{\operatorname{card}(\Omega)} \sum_{i=1}^{\operatorname{card}(\Omega)}-\log p_{i}
$$

with $p_{i}$ the intensity of pixel $i$ and $\operatorname{card}(\Omega)$ the number of pixels $\Omega$.

In addition, assuming that the pixel intensities in each block are distributed following an univariate Gaussian distribution with mean $\mu$ and variance $\sigma, D E_{G}$ is expressed as:

$$
D E_{G}=\log (\sigma)+\frac{1}{\operatorname{card}(\Omega)} \sum_{i=1}^{\operatorname{card}(\Omega)} \frac{\left(p_{i}-\mu\right)^{2}}{2 \sigma^{2}}
$$

We can then compare our proposed similarity measure $D E_{G}$ and the classical one $D E$. To this end, we define two evaluation criteria.

\subsection{Evaluation criteria}

In order to evaluate the quality of registration for each similarity measure, we define two criteria, related to the temporal and spatial dimensions of the method.

Temporal evaluation criterion: We propose a new evaluation criterion based on curve smoothness. The quality of the registration is assessed by the smoothness of the time curve of mean intensities after registration (figure 3). Indeed, after registration, time-intensity curves should reflect the contrast intake only, and not the respiratory motion. Since the presence of kidney in a given axial slice is highly sensitive to respiratory motion, a region of interest (ROI) is defined within the kidney. Inside this ROI, mean intensities $\bar{I}_{t}$ of any image $\left(I_{t}\right)$ before registration and $\bar{J}_{t}$ of the same image after registration are calculated, for each acquisition time $t$ and plotted, see figure 3 for an illustrative example. The two curves are independently filtered by a median time-filter with a window span of 3 points, noted $M\left(I_{t}, 3\right)$ and $M\left(J_{t}, 3\right)$. Therefore, the sum of the absolute difference between the smoothed curve and the original curve can be used as indicator of the smoothness of the enhancement curve. Thus, we define SC as:

$$
S C=\frac{\sum_{t=1}^{T}\left|\bar{J}_{t}-M\left(J_{t}, 3\right)\right|-\sum_{t=1}^{T}\left|\bar{I}_{t}-M\left(I_{t}, 3\right)\right|}{\sum_{t=1}^{T}\left|\bar{I}_{t}-M\left(I_{t}, 3\right)\right|}
$$

The higher $S C$ is, the better quality of registration is. 
Spatial evaluation criterion: the usual metric of $S S D$ was not used in our study since a change in contrast intensity would fool the evaluation. In order to remove variations due to contrast arrival, we decided to used the gradient of the volumes of differences. In order to calculate a spatial criterion on the quality of registrations, we computed two gradient image volumes $G_{I_{t}-I r}$ and $G_{J_{t}-I r}$, and their associated norm $\left\|G_{I_{t}-I_{r}}\right\|$ and $\left\|G_{J_{t}-I_{r}}\right\|$. Eventually, we compute this spatial evaluation criterion, named Gradient Criterion $(G C)$, as:

$$
G C=\frac{1}{T} \sum_{t=1}^{T} \frac{\left\|G_{I_{t}-I_{r}}\right\|-\left\|G_{J_{t}-I_{r}}\right\|}{\left\|G_{I_{t}-I_{r}}\right\|}
$$

$G_{I}$ is defined as the classical gradient of an image $I$ :

$$
G_{I}=\sqrt{\nabla_{X}(I)^{2}+\nabla_{Y}(I)^{2}}
$$

where $\nabla_{X}(I)$ is the gradient of $I$ in $\mathrm{x}$ direction and $\nabla_{Y}(I)$ is the gradient of $I$ in $\mathrm{y}$ direction.

From this gradient image, the $L_{2}$ norm is derived:

$$
\left\|G_{I}\right\|=\sqrt{\sum_{(x, y, z) \in \Omega} G_{I}(x, y, z)^{2}}
$$

This definition is based on the idea that, after registration, the values of difference volume gradients should be lower than before registration. So, a high $G C$ shows a better quality of registration.

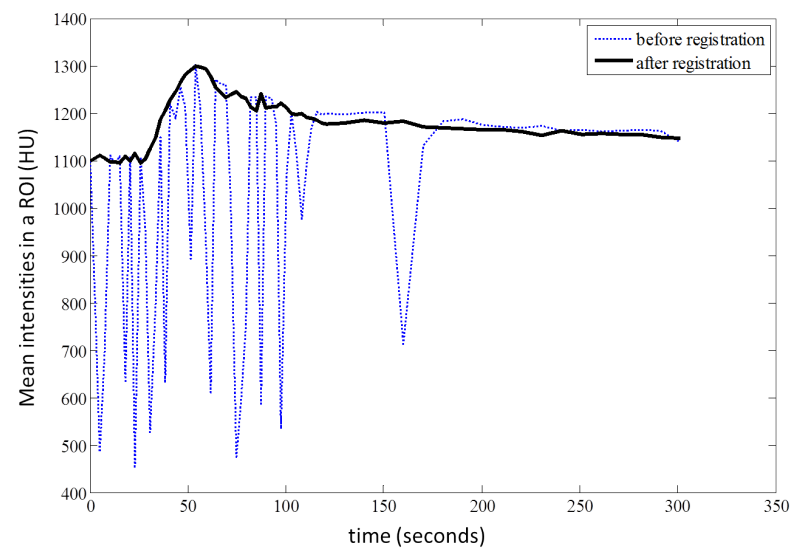

Fig. 3. Mean intensities inside ROI on an axial slice during time of acquisition before registration $\bar{I}_{t}$ (dash line) and after registration $\bar{J}_{t}$ (solid line). 


\section{RESULTS}

Figure 4 shows the results of registration using a checkerboard. After registration, frontiers of organs are better aligned.
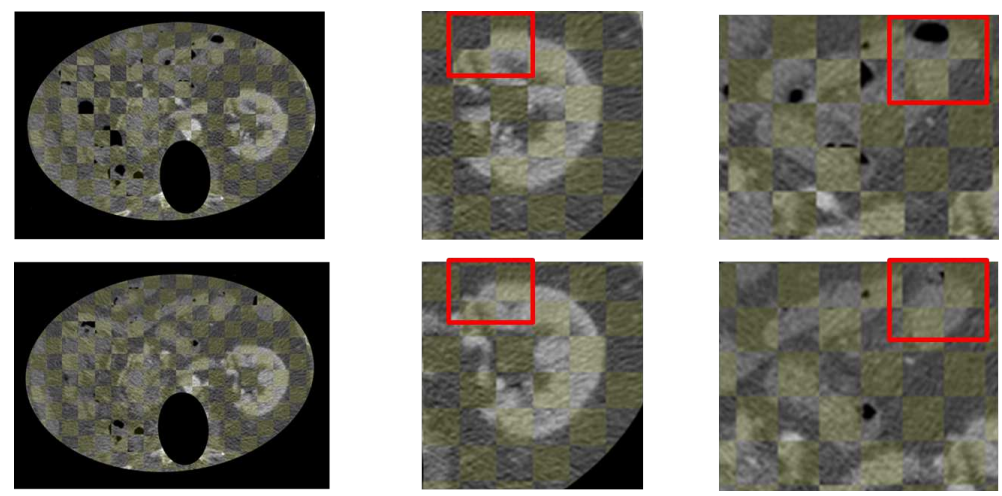

Fig. 4. On the top: before registration; on the bottom: after registration. At left: Checkerboards between image of reference (yellow) and current image (gray). At middle: zoom on kidney, which is better aligned after registration than before, especially in the red squares. At right, zoom on jejunum

After global z-translation, we apply our algorithm of multiresolution blockmatching with $D E$ and our modified similarity measure $D E_{G}$. In figure 5 , results show that our proposed measure $D E_{G}$ is better adapted as similarity measure, according to $S C$ and $G C$ criteria. Note that we tested the assumption of gaussian distribution of pixel intensities in each block. It obviously depends on the size of blocks and their location (blocks that cover two different regions of the image have less normal distribution than blocks that include only homogeneous regions). Our algorithm was nevertheless robust even for blocks that did not verified this assumption.

\section{CONCLUSION}

We have presented a full setup to provide automatic registration of 4D DCE-CT sequences, using an algorithm that consists of global z-translation and multiresolution blockmatching. One main challenge, related to contrast arrival, has been specifically evaluated in the step of multi-resolution block-matching. In this part, we presented an adapted similarity measure (DEG), which was found to be more adapted to registration on free-breathing abdominal $4 \mathrm{D}$ contrast enhanced $\mathrm{CT}$ than $D E$.

Our mid-term objective is to provide a prototype that could be used by clinicians. Therefore, the computation time was one of our main constraints. Our method is relatively efficient. To register one image volume $(512 * 512 * 22)$, it takes about 3 seconds (Intel(R) Core(TM) i5-2450M CPU, 2,5 GHz). This first result is encouraging for a future clinical use. 

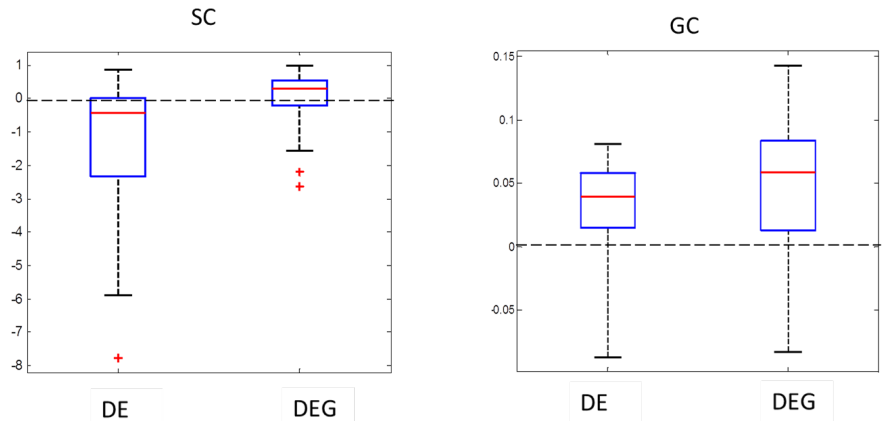

Fig. 5. Values of the two criteria, namely intensity curve smoothness SC (left) and gradient criterion GC (right) for the two similarity measures $D E$ and $D E_{G}$. These plots show that the proposed similarity measure $D E_{G}$ improves the evaluation criteria on the all 39 data acquisitions.

A possible further refinement of our work could be to consider changing of similarity measures with time, according to the diffusion phases of the contrast.

\section{References}

1. Koh, T., et al.: Dynamic contrast-enhanced ct imaging of hepatocellular carcinoma in cirrhosis: feasibility of a prolonged dual-phase imaging protocol with tracer kinetics modeling. Eur Radiol 19(5), 1184-1196 (May 2009)

2. Li, Z., Caan, M., Ziech, M., Stoker, J., van Vliet, L., Vos, F.: 3d non-rigid motion correction of free-breathing abdominal dce-mri data. In: Yoshida, H., Sakas, G., Linguraru, M. (eds.) Abdominal Imaging. Computational and Clinical Applications, Lecture Notes in Computer Science, vol. 7029, pp. 44-50. Springer Berlin / Heidelberg (2012), 10.1007/978-3-642-28557-8-6

3. Rodrigues, E.P., et al.: Evaluation of similarity measures in contrast enhanced echocardiography motion detection and registration. In: Proc. Computers in Cardiology. pp. 773-776 (2009)

4. Romain, B., Lucidarme, O., Dauguet, J., Mul, S., Souedet, N., Chenoune, Y., Guibal, A., Delzescaux, T., Frouin, F.: Registration and functional analysis of ct dynamic image sequences for the follow-up of patients with hepatic tumors undergoing antiangiogenic therapy. IRBM 31(5?6), 263 - 270 (2010)

5. Romain, B., et al.: Optimisation of ct reconstruction for the registration of ct liver perfusion sequences. In: Proceedings of SPIE. vol. 8314(1) (Feb 2012)

6. Tzovaras, D., et al.: Evaluation of multiresolution block matching techniques for motion and disparity estimation. Signal Processing: Image Communication 6(1), 59 - 67 (1994)

7. Wollny, G., Ledesma-Carbayo, M.J., Kellman, P., Santos, A.: Exploiting quasiperiodicity in motion correction of free-breathing myocardial perfusion mri 29(8), 15161527 (2010) 